\title{
Formation au raisonnement clinique : perspectives d'utilisation des cartes conceptuelles
}

\section{Clinical reasoning learning: concept mapping as a relevant strategy}

\section{Anne DEMEESTER ${ }^{1}$, Dominique VANPEE ${ }^{2}$, Claire MARCHAND ${ }^{3}$ et Chantal EYMARD $^{1}$}

1 Département des Sciences de l'Éducation, Université de Provence, Unité mixte de recherche «Apprentissage Didactique Éducation Formation » (UMR-ADEF), Marseille, France

2 Institut de Recherche Santé et Société, Université Catholique de Louvain, Louvain la Neuve, Belgique

3 Laboratoire de pédagogie des sciences de la santé, Unité Propre de Recherche de l'Enseignement Supérieur UPRES EA 3412, Université de Paris 13, Bobigny, France

Manuscrit reçu le 31 juillet 2009; commentaires éditoriaux formulés aux auteurs le 22 février 2010; accepté pour publication le 16 juillet 2010

\section{Mots clés : \\ Raisonnement clinique ; cartes conceptuelles ; organisation des connaissances ; formation en sciences de la santé}

Keywords: Clinical reasoning;
Résumé - Contexte : Le raisonnement clinique développé par les professionnels de santé est une manifestation de leur compétence à résoudre les problèmes posés par les patients. La littérature fait état d'un certain nombre de recommandations permettant aux formateurs en sciences de la santé d'adopter des stratégies pédagogiques pour faciliter l'apprentissage du raisonnement clinique et développer son évaluation. Ces enseignants cliniciens doivent être capables d'expliciter aux apprenants leurs connaissances et leurs actions, de rendre visible et accessible le cheminement et le produit de leurs processus mentaux. Ils doivent également être capables d'identifier, chez les étudiants, les connaissances mobilisées et leurs mises en lien, la nature et le résultat des processus inférentiels mis en œuvre. Les cartes conceptuelles possèdent des propriétés susceptibles d'aider les formateurs dans ces tâches; cependant une recherche bibliographique avancée ne révèle pas leur utilisation dans la formation au raisonnement clinique, alors qu'elles sont par ailleurs utilisées en sciences de la santé, notamment en éducation thérapeutique, pour mettre au jour les connaissances et représentations. Objectifs : Identifier l'utilisation actuelle des cartes conceptuelles en sciences de la santé; repérer leur potentiel d'utilisation pour l'enseignement du raisonnement clinique. Méthode : Revue critique de la littérature et appel à contribution de chercheurs. Résultats : Aucune étude n'a jusqu'ici associé raisonnement clinique et cartes conceptuelles, mais de récentes publications et des travaux portent un intérêt croissant pour la construction de cartes en tant que stratégie d'apprentissage dans l'exploration de la structure des connaissances, la résolution de problème, et le développement d'une pratique réflexive. Conclusion : Cette étude bibliographique montre le potentiel des cartes conceptuelles pour l'apprentissage du raisonnement clinique et ouvre des pistes de recherche.

Abstract - Context: Clinical reasoning skills developed by health professionals show their ability to resolve health problems. Recent research findings provide recommendations 


$\begin{array}{ll}\text { concept mapping; } & \text { for clinical reasoning acquisition and assessment, which may lead clinical teachers to develop } \\ \text { knowledge } & \text { their own pedagogical strategy. Clinical teachers must be able to make their own knowledge } \\ \text { organization; } & \text { and actions understood explicitly as well as their thought process and results clear and acces- } \\ \text { health sciences } & \text { sible. They must also be able to identify students' depth of knowledge, links interconnecting } \\ \text { education } & \text { knowledge, the nature of their inference process and its results. Concept mapping as a tool } \\ & \text { may help clinical teachers in these tasks. However in-depth bibliographic research shows con- } \\ & \text { cept mapping has not been often used in clinical teaching up to now. Purpose: To identify } \\ & \text { the use of concept mapping and its limits. To show compatibility between concept mapping } \\ \text { and recommendations for clinical reasoning training in health sciences. Method: Critical re- } & \text { view of literature and researchers' contributions. Results: Only a few studies link clinical } \\ & \text { reasoning and concept mapping, but recent papers and current research tends to show a grow- } \\ & \text { ing interest in the cartography of knowledge, particularly in problem solving training, critical } \\ & \text { thinking development, and in exploring knowledge structures. Conclusion: This paper shows } \\ & \text { concept mapping may be a relevant and meaningful strategy for clinical teachers concerned } \\ & \text { with clinical reasoning training and gives rise to future research avenues. }\end{array}$

\section{Introduction}

Les professionnels de santé recourent quotidiennement au raisonnement clinique ( $\mathrm{RC})$, ce processus étant notamment sollicité dans le cadre de la résolution de problèmes de santé complexes posés par les patients. Former au raisonnement clinique est une mission difficile pour les enseignants cliniciens, le raisonnement relevant d'une activité mentale qui doit être explicitée pour devenir accessible dans le cadre d'activités d'enseignement et d'apprentissage.

La présente étude constitue la première étape d'une recherche visant à explorer le potentiel d'utilisation de la cartographie des connaissances pour enseigner, apprendre ou évaluer le raisonnement clinique en sciences de la santé. Elle trouve sa légitimité dans les deux propositions suivantes : d'une part, il a été montré que la performance du raisonnement clinique est étroitement tributaire de la qualité de l'organisation des connaissances dans la mémoire à long terme et de la manière dont ces connaissances sont reliées entre elles sous forme de réseaux ${ }^{[1]}$. D'autre part, par les processus cognitifs qu'elle implique, la construction de cartes conceptuelles (CC) - type de cartographie cognitive le plus utilisé et le mieux documenté par des travaux de recherche éducation en sciences de la santé - est une activité de nature à favoriser l'organisation en profondeur des connaissances (liens, niveaux hiérarchiques,...) ${ }^{[2]}$.
Dès lors, on peut faire l'hypothèse que le recours aux $\mathrm{CC}$ dans le cadre des activités d'enseignement et d'apprentissage du RC s'avère efficient.

\section{Problématique}

Le raisonnement clinique

\section{Des stratégies plurielles}

Le développement de la psychologie cognitive a permis de comprendre comment l'homme organise et transforme les informations qu'il reçoit et comment il infère, c'est-à-dire forme de nouvelles connaissances à partir de ces informations. Le polymorphisme du raisonnement humain justifie qu'aient été élaborées des typologies des modes de raisonnement, affinées au fil du temps et des recherches. Ainsi, selon Richard ${ }^{[3]}$, coexisteraient des raisonnements à finalité épistémique (induction, déduction), des raisonnements pour l'action (résolution de problème) et le raisonnement analogique. Parallèlement, des études ont été menées dans le champ particulier de la santé, pour comprendre le raisonnement clinique, mécanisme intellectuel développé par les praticiens. Les travaux publiés concernent quasi exclusivement d'une part, les médecins et, d'autre part, les activités de résolution de problèmes diagnostiques ou thérapeutiques, le terme de raisonnement 


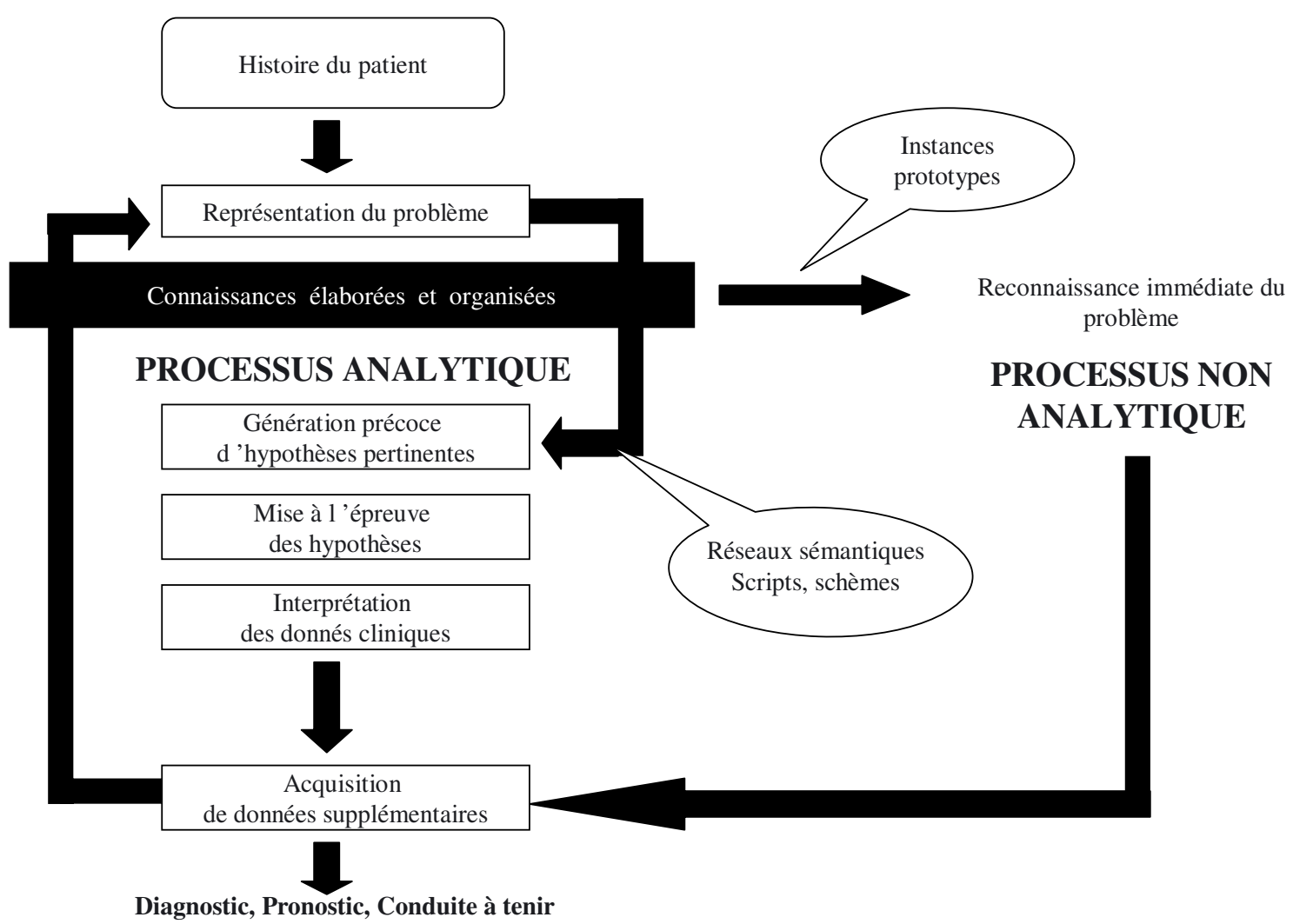

Fig. 1. Raisonnement médical : processus analytique et non analytique, adapté d'après M. Nendaz et al. ${ }^{[9]}$.

médical (RM) se substituant souvent à celui de RC pour désigner l'activité de résolution de problème clinique $^{[4,5]}$. En ce domaine, l'hétérogénéité de la littérature témoigne à la fois de la complexité des processus inférentiels mis en œuvre et de la difficulté d'accéder à l'activité cognitive des sujets et de la rendre explicite ${ }^{[6]}$. Mais plus largement, l'ensemble des professionnels de santé développe au quotidien une ou plusieurs formes de raisonnement, même si les patients ne présentent pas exclusivement des problèmes d'ordre diagnostique ou thérapeutique. À titre d'exemple, une étude exploratoire réalisée auprès de vingt-deux sages-femmes praticiennes a montré que ces dernières développaient des raisonnements de type hypothético-déductif mais également dialectique, voire herméneutique, en fonction des situations cliniques contrastées qui leur étaient présentées ${ }^{[7]}$. Dans cet article, qui concerne les professionnels de santé de différents corps de métier, le terme de RC sera préférentiellement utilisé pour désigner les processus de pensée et de prise de décision, qui permettent au clinicien de conduire les actions les plus appropriées dans un contexte spécifique de résolution de problème de santé ${ }^{[6]}$. Les données les plus récentes permettent de distinguer : les stratégies de RC de forme analytique, conscientes et contrôlées, et les stratégies de RC non analytiques, inconscientes et automatisées ${ }^{[3,8]}$. Selon les contextes et les situations, ces deux modalités coexistent ou se combinent en processus mixtes (figure 1).

Le RC est au cœur de la compétence professionnelle des cliniciens, définie comme un savoir agir complexe acquis et évolutif, s'appuyant sur la mobilisation et la combinaison efficaces de ressources personnelles et externes, pertinentes dans un champ d'expertise particulier ${ }^{[9]}$. La compétence est attachée à une personne ou un collectif dans la 
réalisation d'une activité ${ }^{[10]}$. Selon Chamberland et Hivon, l'enseignement clinique dans les curricula traditionnels de formation en santé est le plus souvent informel, aléatoire et implicite, l'étudiant étant supposé acquérir la compétence clinique en osmose avec les enseignants cliniciens ${ }^{[11]}$.

Ces programmes s'inscrivent désormais de plus en plus dans la perspective d'une approche par compétences et, dans ce cadre, les différentes dimensions de la compétence clinique sont clairement explicitées en tant que finalités visées par le programme. Les formateurs en sciences de la santé doivent donc considérer le RC comme l'une des compétences professionnelles à acquérir. Ceci implique pour les formateurs que, en tant que modèles de rôles, ils soient en mesure d'expliciter leurs connaissances et leurs actions et de rendre visible et accessible le résultat de leur RC, ainsi que le cheminement de leurs processus mentaux ${ }^{[11]}$, étant admis que, d'un expert à l'autre, ce cheminement dans le processus de résolution de problème peut différer ${ }^{[12]}$. Ils doivent aussi être capables d'identifier les connaissances mobilisées par les étudiants, ainsi que les mises en lien que ces derniers parviennent ou non à faire entre ces connaissances.

Au cours des deux dernières décennies et parallèlement aux études menées auprès des praticiens, la recherche en éducation médicale s'est intéressée à l'apprentissage du RC et à son évaluation. Des recommandations ont été publiées pour favoriser le développement de stratégies pédagogiques fondées sur des données probantes et optimiser les pratiques de formation. Certaines de ces recommandations visent à faciliter l'apprentissage du $\mathrm{RC}^{[6,8,11]}$ (tableau I, colonne gauche), d'autres concernent son évaluation $^{[12]}$ (tableau II, colonne gauche).

\section{Rôle de la mémoire dans le raisonnement clinique}

Dans une perspective cognitiviste, les activités mentales telles que le RC ne peuvent être comprises qu'au travers de la distinction entre mémoire de travail (MDT) et mémoire à long terme (MLT) et de la compréhension de leurs échanges et interactions ${ }^{[13]}$.
La MDT permet le traitement conscient de l'information pendant la réalisation d'une activité cognitive complexe (comme le raisonnement ou la résolution de problème clinique) ${ }^{[14]}$. La MLT est le lieu d'encodage, de stockage et de rappel de données, dites informations internes du sujet. C'est également le siège de la mémoire sémantique, essentielle à toute activité mentale complexe telle que le $\mathrm{RC}$ et qui permet la compréhension d'un énoncé, d'un problème, d'une situation. Les informations internes du sujet stockées en MLT - les connaissances antérieures -, permettent, lorsqu'elles sont «activées » en MDT, de traiter les informations externes (énoncé et situation), de leur donner du sens et d'élaborer en MDT une représentation sémantique circonstancielle adaptée à la résolution du problème (encore nommée occurrence). Cette étape de représentation est déterminante pour l'étape de résolution $^{[15]}$. En pratique, lorsqu'un patient soumet un problème de santé au clinicien, ce dernier opère une transformation sémantique qui lui permet d'élaborer une représentation du problème, de lui donner une signification médicale, de proposer des hypothèses diagnostiques et de les comparer par des mises en lien cohérentes. Selon les travaux de Bordage ${ }^{[16]}$ cette même transformation sémantique est opérée par les étudiants placés en situation clinique. La production d'inférences à visée diagnostique, processus de résolution de problème le plus utilisé en médecine, s'appuie soit sur des inférences explicites (raisonnement hypothético-déductif) pour lesquelles le raisonnement se construit pas à pas en MDT ${ }^{[3]}$, soit sur des inférences immédiates déclenchées de façon automatique et augmentant avec l'expérience clinique $^{[6]}$ (reconnaissance de formes - pattern recognition -, activation d'instances ${ }^{[17]}$ ou de prototypes $^{[18]}$ stockés en MLT).

\section{Cartographie des connaissances}

et cartes conceptuelles

\section{Cartographie des connaissances}

La cartographie conceptuelle (concept mapping), développée par Novak ${ }^{[2,23]}$ trouve ses fondements 
Tableau I. Mise en correspondance des recommandations pour l'apprentissage du raisonnement clinique (RC) et propriétés des cartes conceptuelles, à partir des données de la littérature.

\begin{tabular}{|c|c|}
\hline $\begin{array}{l}\text { Recommandations pour l'apprentissage du } \\
\text { raisonnement clinique (RC) }\end{array}$ & Propriétés des cartes conceptuelles \\
\hline $\begin{array}{l}\text { Partager une représentation conceptuelle } \\
\text { commune et explicite du RC entre formateurs } \\
\text { et avec les étudiants }{ }^{[11]}\end{array}$ & $\begin{array}{l}\text { Mettre en évidence les représentations } \\
\text { mentales (des uns et des autres) et les } \\
\text { comparer (analyse des cartes) }^{[23]}\end{array}$ \\
\hline $\begin{array}{l}\text { Favoriser l'apprentissage du RC tout au long } \\
\text { du cursus, aussi précocement que possible, et } \\
\text { au travers de situations cliniques spécifiques }{ }^{[1]}\end{array}$ & $\begin{array}{l}\text { Élaborer des cas problèmes }{ }^{[33]} \\
\text { Travailler en groupe (négocier le sens) ou } \\
\text { individuellement }{ }^{[33]}\end{array}$ \\
\hline $\begin{array}{l}\text { Établir des liens avec les connaissances } \\
\text { antérieures }{ }^{[5,8]}\end{array}$ & \multirow{4}{*}{$\begin{array}{l}\text { Favoriser le regroupement de connaissances, } \\
\text { leurs mise en lien et en réseaux }{ }^{[26]} \\
\text { Rendre les connaissances plus facilement } \\
\text { utilisables }^{[26]} \\
\text { Mettre en évidence les connaissances et mises } \\
\text { en lien }{ }^{[33]}\end{array}$} \\
\hline $\begin{array}{l}\text { Favoriser l'organisation et l'activation des } \\
\text { connaissances }{ }^{[5]}\end{array}$ & \\
\hline $\begin{array}{l}\text { Favoriser l'usage des processus analytiques et } \\
\text { non analytiques }{ }^{[5,8]}\end{array}$ & \\
\hline $\begin{array}{l}\text { Développer des liens explicites entre les } \\
\text { concepts }^{[8]}\end{array}$ & \\
\hline $\begin{array}{l}\text { Faciliter le raisonnement hypothético-déductif } \\
\text { (processus analytique) }{ }^{[5]}\end{array}$ & $\begin{array}{l}\text { Générer des hypothèses }{ }^{[64]} \\
\text { Organiser les connaissances et générer des } \\
\text { hypothèses explicatives }{ }^{[33]} \\
\text { Extérioriser la pensée } \\
\text { Aider à penser dans différentes directions }{ }^{[28]}\end{array}$ \\
\hline $\begin{array}{l}\text { Exposer les apprenants à la résolution de } \\
\text { problèmes complexes }{ }^{[8]}\end{array}$ & $\begin{array}{l}\text { Assimiler de nombreux concepts et relations } \\
\text { complexes }^{[33]} \\
\text { Dévoiler les connaissances employées dans } \\
\text { un contexte de performances complexes } \\
\text { Aider à la résolution de problèmes } \\
\text { complexes }^{[44]}\end{array}$ \\
\hline $\begin{array}{l}\text { Favoriser le transfert des connaissances }{ }^{[5]} \\
\text { Ne pas séparer le développement de stratégies } \\
\text { de RC et l'acquisition des compétences cliniques }{ }^{[11]}\end{array}$ & $\begin{array}{l}\text { Faciliter l'utilisation des connaissances dans } \\
\text { de nouveaux contextes }{ }^{[26]} \\
\text { Augmenter la compréhension et améliorer les } \\
\text { capacités de résolution de problème (en } \\
\text { biologie et chimie) }{ }^{[33]}\end{array}$ \\
\hline $\begin{array}{l}\text { Donner aux étudiants la possibilité de révéler } \\
\text { leurs erreurs idiosyncrasiques }\end{array}$ & $\begin{array}{l}\text { Noter les erreurs, les omissions et les } \\
\text { conceptions erronées } \\
\text { Révéler les conceptions erronées et faire } \\
\text { apparaître le caractère contextuel et les } \\
\text { structures explicatives sous-jacentes }{ }^{[33]} \\
\text { Développer la pensée critique }{ }^{[55-61]}\end{array}$ \\
\hline
\end{tabular}


Tableau II. Mise en correspondance des recommandations pour l'évaluation du raisonnement clinique (RC) et des propriétés des cartes conceptuelles, à partir des données de la littérature.

\begin{tabular}{|c|c|}
\hline $\begin{array}{l}\text { Recommandations pour l'évaluation du } \\
\text { raisonnement clinique (RC) })^{[12]}\end{array}$ & Propriétés des cartes conceptuelles \\
\hline $\begin{array}{l}\text { Avoir recours à plusieurs outils } \\
\text { complémentaires car le RC est } \\
\text { multidimensionnel }\end{array}$ & $\begin{array}{l}\text { Évaluation formative }{ }^{[33]} \text {, évaluation } \\
\text { diagnostique }{ }^{[29]} \\
\text { Comparer les cartes réalisées par des étudiants } \\
\text { à celle réalisée par un expert } \\
\text { Support pour questionner les étudiants sur } \\
\text { leurs connaissances } \\
\text { Proposer des cartes à trous } \\
{[36]}\end{array}$ \\
\hline $\begin{array}{l}\text { Sélectionner des situations réelles, des cas } \\
\text { courts plutôt qu'un cas long }\end{array}$ & Élaborer des cas problèmes ${ }^{[33]}$ \\
\hline $\begin{array}{l}\text { S'attacher plus à la tâche cognitive effectuée } \\
\text { qu'au format de l'évaluation }\end{array}$ & $\begin{array}{l}\text { Visualiser comment les apprentissages se } \\
\text { réalisent }{ }^{[30]} \\
\text { Apprécier la façon dont l'apprenant construit } \\
\text { et organise ses connaissances }{ }^{[32]} \\
\text { Mettre en évidence les processus de pensée } \\
\text { Permettre de représenter et d'exprimer des } \\
\text { habiletés intellectuelles }{ }^{[26]}\end{array}$ \\
\hline
\end{tabular}

dans les recherches menées en psychologie cognitive dès les années soixante par Ausubel ${ }^{[19]}$, qui souligna notamment la différence entre un apprentissage par cœur (rote learning) et un apprentissage signifiant (meaningful learning). Les principes de la cartographie de la connaissance (cartography of cognition) ont été décrits par Wandersee ${ }^{[20]}$. La littérature sur le sujet est très abondante, comme le soulignent Nesbit et Adesope, dans une meta-analyse récente, faisant état de 500 références disponibles entre 1985 et 2006, et montrant un intérêt croissant des chercheurs en éducation à partir de $1997^{[66]}$. Il existe une certaine confusion dans la littérature, liée à l'existence de plusieurs formes de représentations graphiques proches les unes des autres. La carte conceptuelle (CC) est probablement la plus connue dans le champ des sciences de la santé car elle a fait l'objet de nombreuses recherches et publications, notamment en éducation thérapeutique de patients ${ }^{[21]}$. D'autres formes de cartes existent également $^{[7]}$ : la carte de connaissances (avec liste préétablie de concepts et relations), les réseaux sémantiques (avec liens bidirectionnels, sans organisation hiérarchique), les cartes mentales (avec concept central, sans organisation hiérarchique), les cartes cognitives (avec relations causales, sans explicitation des liens). Ces différentes constructions ne partagent pas de grammaire commune.

La présente étude se limite aux CC. Souvent présentée comme un outil, une CC est en fait le produit d'une activité de construction ${ }^{[24]}$ mettant au jour des connaissances sous la forme d'une représentation hiérarchisée d'un ensemble de concepts et de liens de sens qu'un individu établit entre ces différents concepts ${ }^{[2,22]}$. Considérée comme indépendante du domaine, la construction de CC est utilisée en tant que stratégie d'apprentissage et de représentation des connaissances ${ }^{[23]}$. Élaborer une $\mathrm{CC}$ consiste à créer une représentation graphique prenant la forme d'un réseau de nœuds et d'arcs, où les nœuds représentent les concepts et les arcs, les liens définis entre les concepts. Une connaissance se traduit par deux concepts reliés par une flèche qui figure ce lien de sens explicitement défini. 


\section{Clarification sémantique : représentations, concepts et connaissances}

Les représentations circonstancielles (ou occurrences ${ }^{[13]}$ ) sont des constructions faites dans un contexte particulier et à des fins spécifiques. Elles sont directement liées à la tâche et à la nature des décisions à prendre et sont produites en MDT. Les représentations ont un caractère transitoire et peuvent, une fois la tâche terminée, être remplacées par d'autres représentations liées à d'autres tâches. D'un point de vue cognitif, et en tant qu'activité de résolution de problème, le RC est un processus de construction de représentations circonstancielles, directement liées au but et à la nature de la tâche et dépendant de l'organisation des connaissances en MLT.

Certaines des représentations construites ne sont pas oubliées mais stockées dans la MLT et susceptibles d'être rappelées, comme lors du transfert analogique décrit par Richard ${ }^{[3]}$. Ces reconnaissances spontanées constituent la part non analytique du raisonnement ${ }^{[5,8]}$.

Les concepts sont les supports de la transmission de sens par le langage. Un concept (concret ou abstrait) est désigné par un nom et a un sens. Par exemple, nuage est un concept concret et idée un concept abstrait. Les concepts sont stockés en MLT des sujets et sont reliés entre eux par une multiplicité de liens appelés réseaux sémantiques ou réseaux conceptuels.

Les connaissances (ou représentations types ${ }^{[13]}$ ) sont des constructions également stockées dans la MLT. Elles sont stables et peu dépendantes de la tâche à réaliser mais susceptibles d'être modifiées $^{[24]}$. Elles se répartissent classiquement en connaissances déclaratives (relatives aux concepts), procédurales (relatives aux procédures) et conditionnelles (relatives au contexte) ${ }^{[20]}$. Les connaissances sont générales (appropriées à un vaste ensemble de tâches) ou spécifiques (étroitement liées à des contenus disciplinaires particuliers). Lorsque l'apprenant met en lien une information nouvelle avec son réseau de connaissances préalables, il réorganise ses connaissances. Cette réorganisation témoigne de l'apprentissage ${ }^{[25]}$. Par une étude menée auprès de cliniciens experts et novices, Bordage a montré l'importance de l'organisation des connaissances dans la capacité à résoudre les problèmes cliniques $^{[1]}$. Il décrit quatre formes d'organisation des connaissances qu'il qualifie de réduites, dispersées, élaborées ou compilées en réseaux richement interconnectés. Toujours selon Bordage, plus les connaissances des praticiens sont organisées et plus elles sont reliées entre elles sous forme de réseaux, meilleures sont les capacités à résoudre des problèmes et à prendre des décisions. Les conclusions de ces travaux contribuent à faire penser que le développement de stratégies d'enseignement visant à mieux organiser les connaissances serait susceptible d'améliorer le raisonnement clinique. Cette hypothèse est également émise par Coderre et al. ${ }^{[26]}$ dans le cadre d'une recherche qualitative auprès de 91 étudiants, qui visait à confronter l'organisation de leurs connaissances avec leur performance diagnostique. Ils concluent que, la performance diagnostique étant corrélée à l'organisation des connaissances, améliorer la structure des connaissances devrait améliorer la performance diagnostique.

\section{Propriétés des cartes conceptuelles}

Les CC présentent de nombreuses propriétés dans des domaines didactiques variés ${ }^{[66]}$. Les CC permettent de rendre l'apprentissage signifiant pour l'apprenant, d'extérioriser les connaissances du sujet à partir desquelles de nouvelles connaissances pourront être acquises, et de mettre en évidence ses connaissances erronées ou partielles ${ }^{[27,28]}$. Elles permettent aussi de visualiser comment les apprentissages se réalisent par la définition de liens de sens entre les concepts ${ }^{[29]}$. Construites individuellement ou collectivement ${ }^{[30]}$, les cartes permettent notamment d'organiser les connaissances et de faciliter leur mémorisation mais elles permettent aussi l'expression de représentations, concepts et connaissances, susceptibles d'interférer dans la résolution de problème ou la prise de décision ${ }^{[24]}$. C'est en premier lieu la lecture de la carte, puis son analyse 
qui donnent accès à ces informations. L'analyse des cartes conceptuelles est à la fois quantitative et qualitative ${ }^{[31-33]}$. D'une part elle dénombre les concepts, leurs niveaux de hiérarchisation et les liens entre ces concepts ; d'autre part, elle s'intéresse à la nature et à l'organisation des connaissances.

Au total, il serait prématuré de développer dès à présent des travaux s'inscrivant dans une logique de démonstration de la validité de notre hypothèse générale, à savoir l'efficience du recours aux cartes conceptuelles dans le cadre d'activités d'enseignement et d'apprentissage du raisonnement clinique. En revanche, de nombreux arguments invitent à documenter de manière méthodique et systématique la viabilité d'une telle piste de recherche. Dans le cadre d'un travail exploratoire et d'une démarche globale d'amélioration des pratiques de formation en sciences de la santé, les objectifs du présent travail sont : a) de faire l'inventaire des utilisations actuelles des CC dans le cadre de la formation des professionnels en sciences de la santé ; b) d'identifier leur potentiel d'utilisation pour l'enseignement et l'apprentissage du raisonnement clinique.

\section{Méthodes}

Nous avons, d'une part, procédé à une revue méthodique et critique de la littérature scientifique dédiée à la problématique des $\mathrm{CC}$ en éducation des sciences de la santé et, de l'autre, nous avons sollicité la contribution de chercheurs et de personnes ressources sélectionnées ad hoc.

Stratégie de recherche documentaire

Les articles publiés et travaux en cours associant CC et formation au RC en sciences de la santé ont été recherchés, ainsi que les articles permettant d'identifier des éléments en faveur du recours des cartes pour la formation au RC. La recherche porte sur neuf années de publications (2000 à 2008).

\section{Les publications}

Les mots-clés utilisés pour la recherche bibliographique sont : cartes conceptuelles, organisation des connaissances, raisonnement clinique, raisonnement médical, et leur traduction en langue anglaise : concept mapping, concept map, knowledge organization, medical reasoning, clinical reasoning. Plusieurs associations de mots-clés ont été testées afin de recenser les articles susceptibles de répondre aux objectifs fixés. Notre recherche documentaire a d'abord exploité la base de données Medline. Elle a ensuite été étendue au domaine des sciences humaines afin de recenser les publications en sciences de l'éducation où sont répertoriés les articles en éducation médicale ou éducation en sciences de la santé de langue anglaise; ont été utilisées l'interface d'interrogation Ovid (recherche fédérée dans Pascal, Eric et Francis), la base de données Web of Knowledge et le moteur de recherche spécialisé Google scholar. Les articles ont été sélectionnés selon la méthode recommandée par l'agence nationale d'évaluation en santé (ANAES) ${ }^{[34]}:$ recherche à l'aide des mots-clés, première sélection des articles à l'aide du titre, seconde sélection à l'aide du résumé, troisième sélection après lecture de l'article. Une recherche de proche en proche a été associée, à partir des articles sélectionnés.

\section{La littérature grise}

Un recensement des thèses (catalogue collectif des bibliothèques universitaires françaises - SUDOC), des mémoires répertoriés en bibliothèque universitaire pour l'obtention de diplôme universitaire, de master en éducation médicale et du diplôme de cadre sage-femme, a été réalisé.

Appel à contribution de chercheurs et personnes ressources

Une liste de personnes connues pour développer des recherches ou un intérêt particulier dans le champ 
du raisonnement clinique ou des cartes conceptuelles a été établie à partir d'abstracts de communications scientifiques et de proche en proche. $\mathrm{Du}$ fait de la dispersion géographique de ces personnes (cinq pays et deux continents), former un groupe de discussion ou procéder à des entretiens individuels semi-structurés n'était pas possible. Un courrier électronique accompagné d'un court questionnaire a été adressé en janvier 2009 à 28 chercheurs ou enseignants et personnes d'appartenance professionnelle différente dans le champ sanitaire. Les questions portaient sur la connaissance des cartes, leur utilisation en sciences de la santé dans et hors le champ du RC et la pertinence d'exploiter les CC dans le cadre de la formation au RC.

\section{Résultats}

\section{Analyse des publications}

À l'aide des mots-clés, 216 références ont pu être trouvées, 74 ont été sélectionnées par leur titre et, après lecture des résumés, 32 articles ont été retenus. Seize contribuent à actualiser les utilisations des CC en sciences de la santé et 16 mettent en évidence des critères de compatibilité entre RC et CC. Aucune étude associant directement $\mathrm{CC}$ et RC n'a été trouvée.

\section{Actualisation des utilisations connues des cartes conceptuelles en sciences de la santé}

Un article publié en novembre 2004 par Marchand et d'Ivernois dans la présente revue a décrit les différents usages des CC dans les formations en santé ${ }^{[32]}$. Les références déjà citées par ces auteurs ont été enrichies par une réactualisation de la bibliographie.

\section{Aide à l'apprentissage}

Plusieurs publications confirment que le principal usage des cartes est de favoriser le caractère signifiant de l'apprentissage ${ }^{[35-37]}$. D'autres écrits mettent en évidence la construction progressive de savoirs chez des formateurs en sciences de la santé, sollicités à plusieurs temps de leur parcours de spécialisation en éducation ${ }^{[32]}$, ou chez des étudiants infirmiers ${ }^{[38]}$. La force métacognitive des cartes est également mentionnée ${ }^{[32,39]}$. Les cartes sont notamment utilisées dans la champ de l'éducation thérapeutique des patients atteints de maladie chroniques $^{[40-42]}$.

\section{Aide à la planification}

Initialement proposées par leurs concepteurs, Novak et Gowin, comme aide à la planification des enseignements, cet usage premier des $\mathrm{CC}$ a récemment été transféré en sciences infirmières : planification et évaluation de démarches de soins primaires par des étudiants infirmiers ${ }^{[43]}$, modélisation de plan de soins infirmiers ${ }^{[4]}$, planification de cours et objectifs en santé publique ${ }^{[45]}$.

\section{Évaluation}

Peu d'études portent sur l'usage des cartes dans l'évaluation en sciences médicales, alors qu'elles peuvent, comme le souligne West, explorer l'organisation et l'utilisation des connaissances, identifier des lacunes, des connaissances erronées ou des difficultés de mises en lien entre les concepts ${ }^{[30]}$. Fautes de qualités psychométriques estimées suffisantes ${ }^{[46,47]}$, les cartes répondent plus aux critères de l'évaluation formative ${ }^{[48]}$ ou diagnostique ${ }^{[49]}$ qu'à ceux de l'évaluation sommative. Dans une perspective d'évaluation des compétences, Tardif souligne néanmoins que la $\mathrm{CC}$ constitue un outil puissant à intégrer dans le dossier d'apprentissage ; elle permet aux étudiants de fournir des preuves de leur apprentissage en matière de ressources et en matière de situations de transfert ${ }^{[22]}$.

La récente revue de la littérature, publiée après notre propre étude, confirme ces utilisations ${ }^{[50]}$. 


\section{Potentiel d'utilisation des CC pour l'enseignement du RC}

Seize publications traitant soit des CC, soit du RC, ont contribué à mettre en évidence des critères de compatibilité entre l'activité de construction de cartes et les recommandations émises pour la formation au $\mathrm{RC}^{[6,8,11,12]}$. Recommandations et arguments des auteurs, illustrant le potentiel d'utilisation des $\mathrm{CC}$ cette perspective, ont été mis en correspondance (tableaux I et II).

\section{Analyse de la littérature grise}

Trois thèses traitant des $\mathrm{CC}$ ont été publiées en $2000^{[51]}, 2005$, et $2006^{[23]}$. Seule celle soutenue par Marchand ${ }^{[51]}$ s'inscrit dans le domaine de la santé mais ne concerne pas le RC développé par les apprenants. Plus d'une dizaine de mémoires écrits par des professionnels de santé et traitant du RC ont été recensés. Un seul abordait les CC, dans le domaine de la formation initiale sage-femme ${ }^{[52]}$ sous forme de proposition pour améliorer l'enseignement au RC.

\section{Contribution des chercheurs et des personnes ressources}

Dix-sept questionnaires ont pu être exploités parmi les 28 adressés. Tous les répondants connaissent les $\mathrm{CC}$ (ou variantes); plusieurs les utilisent ou les ont déjà utilisées $(n=11)$, certains dans le but d'explorer les structures du raisonnement clinique $(n=7)$. Treize déclarent avoir déjà pensé associer CC et RC. Les utilisateurs recourent à l'outil de façon empirique, aucune étude sur le sujet n'étant à ce jour soumise à publication. Les domaines d'utilisation déclarés ou potentiels sont par ordre de fréquence : l'exploration des connaissances et représentations ( $n=16)$, l'évaluation formative $(n=14)$, l'éducation thérapeutique $(n=12)$, l'apprentissage (synthèse d'une formation, d'un cours, d'un cas clinique, fiche de lecture; approche par problème) $(n=12)$, la planification $(n=11)$.

\section{Discussion}

\section{Limites et biais de l'étude}

L'étude comporte des biais d'information et de sélection. Elle se limite aux seules cartes conceptuelles, excluant les autres formes de cartographie cognitive. La recherche bibliographique n'a concerné que les articles disponibles en français et en anglais dans les revues indexées. Le recensement des thèses et mémoires s'est limité aux institutions françaises et ne concernait que les travaux répertoriés en bibliothèque universitaire. Pour l'appel à contribution, seuls des francophones ont été sollicités. Ils étaient connus pour l'intérêt particulier qu'ils portaient soit aux cartes, soit au raisonnement médical.

\section{Compatibilité entre cartes conceptuelles} et formation au raisonnement clinique

L'étude montre que si l'usage des CC tend à se développer en sciences de la santé, très peu d'articles associent $\mathrm{CC}$ et RC. Cependant, les travaux les plus récents soulignent un nouvel intérêt des chercheurs pour la construction de CC dans le cadre de l'apprentissage de la résolution de problèmes cliniques. Rendas et al. ont mené une étude qualitative incluant 14 étudiants en médecine, dont le protocole de recherche associait apprentissage par problème (problem-based learning-PBL) et construction de $\mathrm{CC}^{[35]}$. Au total, 36 cartes ont été élaborées, permettant aux auteurs de constater la progression des étudiants en physiopathologie et de conclure sur la complémentarité des deux techniques, les cartes potentialisant selon eux, les effets de l'approche par problème. Cette stratégie pédagogique a par ailleurs satisfait l'ensemble des étudiants concernés. Une étude proposant la construction de CC en biologie et chimie montre que ces dernières améliorent la compréhension d'un problème et facilitent sa résolution ${ }^{[33]}$. Massart et al. ${ }^{[53]}$ proposent l'usage des CC dans un dispositif d'apprentissage à la résolution de problèmes complexes pour «faire émerger l'organisation cognitive d'étudiants en médecine 
et évaluer qualitativement et quantitativement : les actions de recours pluridisciplinaires lors de la résolution de problème écrit, la dimension organisationnelle et la complexité de structuration des connaissances ». Comte ${ }^{[52]}$ propose d'introduire les cartes conceptuelles dans la formation au RC des sagesfemmes : «Dès la première année d'étude, il paraît souhaitable d'enseigner cette méthode utilisant la schématisation aux étudiants car ceux qui l'ont appris, l'utilisent pour la résolution de problèmes et intègrent plus facilement les concepts scientifiques et cliniques de base ». Le rôle des cartes dans le développement de la pensée critique (critical thinking) est décrit spécifiquement dans le domaine des sciences infirmières en Amérique du nord ${ }^{[54-59]}$. Ce courant de recherche a été impulsé par différentes instances supervisant la formation des infirmiers américains (American Association of Colleges of Nursing's en 1998, National League for Nursing Accrediting Commission en 2002). La pensée critique est définie comme un processus intellectuel auto-régulé conduisant à un jugement adapté (process of purposeful, self-regulated judgment), dont les concepts clés sont : interprétation, analyse, évaluation et inférence ${ }^{[60]}$. Cette démarche intellectuelle initialement décrite par Dewey ${ }^{[61]}$ se déroule en cinq temps : la reconnaissance d'un problème, la définition de ce problème, la formulation de solutions hypothétiques, l'anticipation des résultats possibles et la mise à l'épreuve des hypothèses résolutives. La pensée critique, également développée à l'aide du portfolio ${ }^{[12,62]}$, relève d'une pratique réflexive très proche des processus de raisonnement analytique. Si les CC aident au développement de la pensée critique, il est probable qu'elles puissent contribuer au développement des stratégies analytique du RC, tel que le raisonnement hypothético-déductif qui est couramment utilisé dans la démarche diagnostique. Burke et al. soulignent par ailleurs que les cartes peuvent aider les chercheurs à générer des hypothèses et développer de nouvelles théories ${ }^{[63]}$, ce qui laisse supposer l'aide apportée par les CC dans la génération d'hypothèses, étape du raisonnement clinique. La plupart des contributions apportées par les chercheurs et les personnes ressources qui ont été sollicitées corroborent les données de la littérature; toutefois d'autres perspectives de recherche et d'utilisation apparaissent. Dans le cadre de l'exploration des connaissances, des chercheurs de différentes disciplines en santé de l'université de Montréal travaillent actuellement sur un projet de modélisation du raisonnement clinique. Le groupe est assisté d'un designer pédagogique spécialisé dans l'analyse de contenu auprès d'experts, dans et hors le champ de la santé. Ce travail de modélisation se fait à l'aide de MOT Plus, un logiciel permettant la mise en forme de cartes conceptuelles avec l'application d'une grammaire particulière (quatre formes de connaissances et sept types de liens entre ces connaissances) ${ }^{[64]}$. Il en résulte que la cartographie cognitive obtenue est plus facile à lire et que le logiciel contribue à la fidélité de son interprétation, se révélant ainsi être un outil prometteur. Les autres commentaires libres suggèrent, sur la base d'un usage empirique des cartes en supervision clinique, qu'il y aurait un lien entre le recueil de données ciblées et la carte obtenue. Par ailleurs, deux chercheurs proposent la sémantique structurale comme cadre conceptuel pour l'analyse de cartes.

\section{Limites des cartes conceptuelles}

Construire une carte nécessite d'avoir bénéficié d'une séquence d'initiation préalable. Co-construire une carte avec l'étudiant demande du temps au formateur. Ces contraintes constituent probablement des freins à une plus large diffusion et utilisation de la technique. Mais la principale difficulté des CC réside dans leur analyse car il n'existe pas à ce jour de consensus ou de grammaire commune qui objective cette étape. De plus, les cartes font état de l'organisation des connaissances mais pas de leur utilisation. De nombreuses questions de recherche persistent notamment sur la validité et la fiabilité des $\mathrm{CC}^{[48]}$, ce qui peut conduire, dans une perspective psychométrique, à en restreindre l'usage à des tâches d'évaluation formative ou diagnostique. D'autres instruments, comme les TCS semblent plus appropriés pour évaluer les processus de $\mathrm{RC}^{[65]}$. 


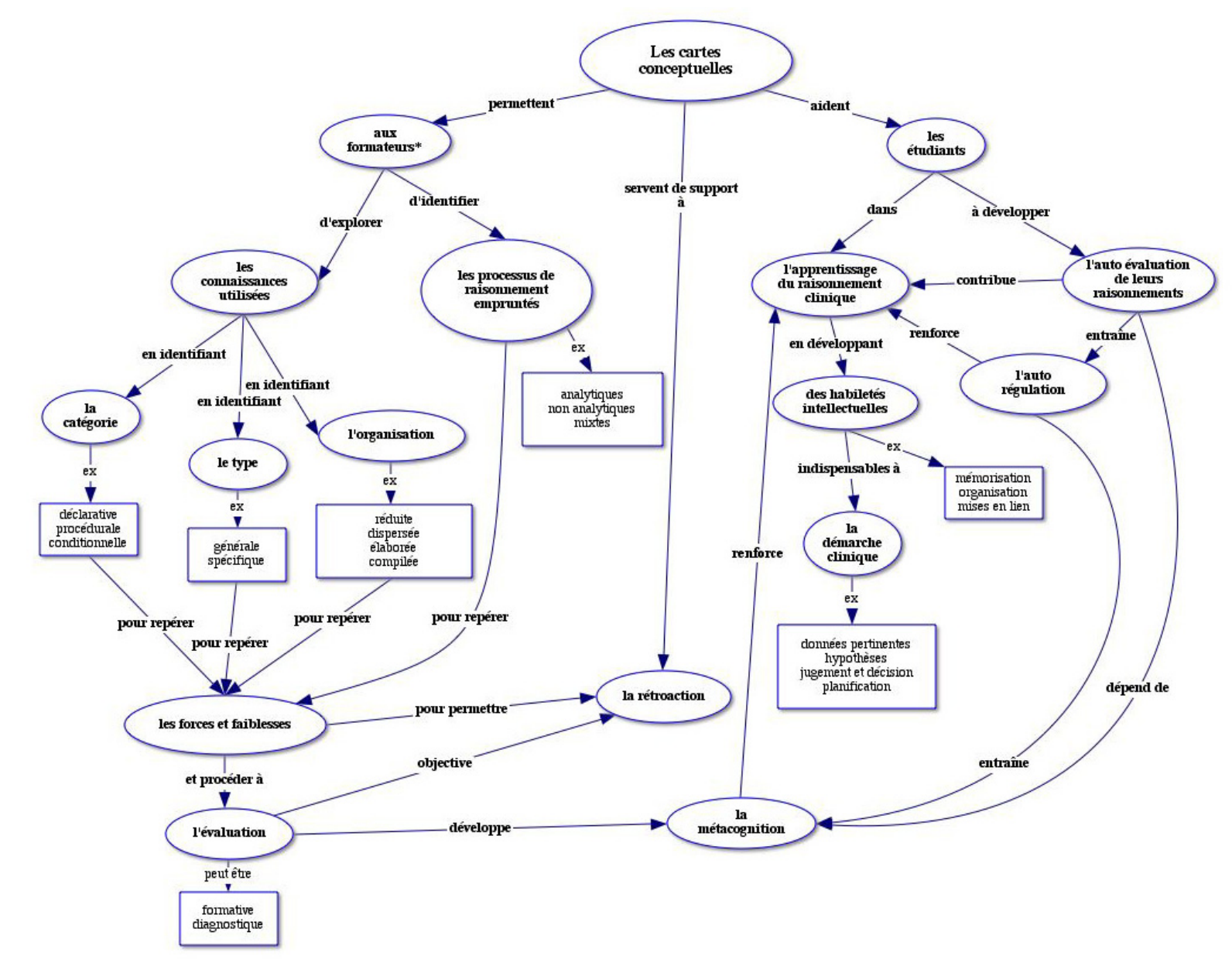

*formateurs : enseignants cliniciens, enseignants chercheurs

Fig. 2. Perspectives d'utilisation des cartes conceptuelles dans la formation au raisonnement clinique.

Perspectives d'utilisation dans le champ de la formation au raisonnement clinique

Concernant la formation au RC, deux axes d'utilisation des CC se profilent et sont représentés ici sous forme d'une CC de synthèse conçue pas les auteurs (figure 2).

Proposer aux étudiants de construire des CC peut servir aux formateurs dans plusieurs cas : a) pour explorer les connaissances des apprenants (catégorie, type, organisation); b) pour identifier les mises en lien entre ces connaissances et les processus de RC qu'ils empruntent; c) pour faire émerger le RC de professionnels, de cliniciens ou d'étudiants, afin de les confronter; d) pour observer l'évolution de l'organisation des connaissances tout au long d'un parcours de formation; e) pour repérer les forces et faiblesses du raisonnement, identifier les lacunes, les erreurs, ... Les CC peuvent être utilisées comme support de feedback en supervision clinique et inciter les étudiants à une pratique réflexive. Les CC aident les étudiants à faire la synthèse de cas cliniques ou à planifier une conduite à tenir mais elles sont susceptibles de les aider à développer un $\mathrm{RC}$ de forme analytique et à cheminer vers la compétence de raisonnement clinique. 


\section{Conclusion}

En s'appuyant sur une revue critique de la littérature et sur les résultats d'un appel à contribution de chercheurs et personnes ressources, ce travail a permis d'une part d'actualiser les données scientifiques disponibles sur l'usage actuel de CC dans les dispositifs de formation en santé et, d'autre part, de mettre en correspondance les recommandations pour la formation au RC avec les propriétés des cartes $\mathrm{CC}$, contribuant ainsi à identifier leur potentiel d'utilisation dans ce domaine.

La cartographie des connaissances a récemment trouvé sa place en supervision clinique notamment pour initier les étudiants à une pratique réflexive dont le déroulement cognitif est comparable à celui du raisonnement clinique de forme analytique. Si quelques auteurs préconisent l'utilisation de CC dans la formation au RC, aucune recherche analytique confirmant leurs propos n'a été publiée sur le sujet. Les CC, présentées par Tardif comme un outil puissant dans la construction des compétences ${ }^{[9]}$, nécessitent à présent d'être éprouvées par la recherche dans le champ spécifique du RC. Plusieurs questions se posent, ouvrant autant de pistes de recherche : la construction de CC facilite-t-elle le développement des processus de raisonnement analytique face à une situation clinique? Les étudiants progressent-ils mieux dans l'acquisition de la démarche clinique lorsqu'ils construisent des CC ? Les sujets qui produisent les cartes conceptuelles les plus élaborées sont-ils les plus performants dans la résolution de problème de santé ${ }^{[48]}$ ?

L'intérêt et la curiosité manifestés par les chercheurs vis-à-vis de cette stratégie cognitive d'une part, et le caractère exclusivement descriptif des rares études publiées, encouragent à poursuivre les investigations. Les résultats de cette approche bibliographique identifient des perspectives de recherche explorant la problématique de l'association $\mathrm{CC}$ et $\mathrm{RC}$, en suggérant que les CC pourraient se révéler pertinentes dans la formation au $\mathrm{RC}$ des professionnels de santé.

\section{Contributions}

Anne Demeester a effectué la revue critique de la littérature, a lancé l'appel à contribution, et a rédigé l'article. Chantal Eymard et Dominique Vanpee sont les deux promoteurs de l'étude. Claire Marchand a saisi la version électronique de la carte conceptuelle à l'aide du logiciel Inspiration ${ }^{\complement}$. Tous les auteurs ont révisé l'article.

\section{Remerciements}

Nous remercions toutes les personnes qui ont contribué à cette étude, en répondant au questionnaire ou en nous communiquant leurs travaux, suggestions, commentaires.

\section{Références}

1. Bordage G. Elaborated knowledge: a key to successful diagnostic thinking. Acad Med 1994;69-11:883-5.

2. Novak JD, Gowin DB. Learning how to learn. New-York: Cambridge University Press, $4^{\mathrm{e}}$ édition, 1989.

3. Richard J-F. Les activités mentales, de l'interprétation de l'information à l'action. Paris : Armand Colin collection U, $4^{\mathrm{e}}$ édition, 2005.

4. Masquelet A. Le raisonnement médical. Paris: PUF Collection Que sais-je ? n³764, 2006.

5. Nendaz M, Charlin B, Leblanc V, Bordage G. Le raisonnement clinique : données issues de la recherche et implications pour l'enseignement. Pédagogie Médicale 2005;6:235-54.

6. Guilbert J-J. Comment raisonnent les médecins, réflexions sur la formation médicale, Genève : Éditions Médecine et Hygiène, 1992.

7. Demeester A. Modes de raisonnement des sages-femmes en situation clinique. Mémoire pour l'obtention du grade de master recherche en sciences de l'éducation. Université de Provence, 2005.

8. Eva KW. What every teacher needs to know about clinical reasoning. Med Educ 2004;39:98-106.

9. Tardif J. L'évaluation des compétences. Montréal : Éditions Chenelière, 2006.

10. Wittorsky R. Analyse du travail et production de compétences collectives, Éditions de l'Harmattan, 1997. 
11. Chamberland M, Hivon R. Les compétences de l'enseignant clinicien et le modèle de rôle en formation clinique. Pédagogie Médicale 2005;6:98-111.

12. Charlin B, Bordage G, Van der Vleuten C. L'évaluation du raisonnement clinique. Pédagogie Médicale 2003;4:42-52.

13. Le Ny JF. Comment l'esprit produit du sens, notions et résultats des sciences cognitives. Paris : Éditions Odile Jacob, 2005.

14. Roulin J-L (Coord.). Psychologie Cognitive Rosny: Édition Bréal, Collection Grand Amphi (2 édition), 2006.

15. Nendaz M, Bordage G. Promoting Diagnostic problem representation, Med Educ 2002;36:760-6

16. Bordage G, Lemieux M. Semantic structures and diagnostic thinking of experts and novices. Acad Med 1991;71:55-61.

17. Schmidt G, Norman G, Boshuizen H. A Cognitive Perspective on Medical Expertise: Theory and Implications. Acad Med 1990;65:611-21

18. Bordage G, Zacks R. The structure of medical knowledge in the memories of medical students and general practitionners: categories and prototypes. Med Educ 1984;18:406-16

19. Ausubel D. Educational psychology. A cognitive view. New York: Holt, Rinehart and Winston, $2^{\mathrm{e}}$ édition, 1978.

20. Wandersee JH. Concept mapping and the cartography of cognition. J Reas Sci Teach 1990;27:923-36.

21. Marchand C, d'Ivernois JF, Slama G, Assal JP, Hivon R. An analysis, using concept mapping, of diabetic patient's knowledge, before and after patient education. Med Teach 2002, 24-1:90-9.

22. Nowak J, Canas A. The theory underlying concept maps and how to construct and use them. Technical report IHMC CmapTols 2006-01 rev 01-2008 [On-line] disponible sur: www.ihmc.us

23. Pudelko B. Étude microgénétique des médiations épistémiques d'un outil informatisé de représentation graphique des connaissances au cours d'une activité de compréhension de texte : proposition pour une approche instrumentale étendue des médiations des outils cognitifs dans l'apprentissage. Thèse de doctorat en psychologie cognitive, Université Paris VIII, 2006.

24. Richard J-F. Les activités mentales, comprendre, raisonner, trouver des solutions. Paris : Armand Colin, 1990.

25. Tardif J. Pour un enseignement stratégique, l'apport de la psychologie cognitive. Montréal : Éditions logiques, 1992, chapitre 1 : Introduction à la psychologie cognitive, 25-86.
26. Coderre S, Jenkins D, Mclaughlin K. Qualitative differences in knowledge structure are associated with diagnostic performance in medical student. Adv Health Sci Educ 2009;14:677-84

27. Iguenane J, Marchand C, d'Ivernois J-F. Les cartes sémantiques, outil de formation. Les politiques sociales : cartes et relations, 1999;26-45.

28. West DC. Critical thinking in graduate medical education, a role for concept mapping assessment? JAMA 2000;284:1105-1110.

29. Marchand C. Intérêt des cartes sémantiques dans l'éducation du patient, Bull. Éduc. du Patient 1997,16;4:33-6.

30. Tribollet B, Langlois F, Jacquet L. Protocoles d'emploi des cartes conceptuelles au lycée et en formation des maîtres. Montpellier : TREMA - IUFM 2000, 18-61.

31. Marchand C, Gagnayre R. Utilisation de la carte conceptuelle auprès de formateurs en santé pour l'apprentissage de concepts pédagogiques. Pédagogie Médicale 2004;5:13-23.

32. Marchand C, d'Ivernois J-F. Les cartes conceptuelles dans les formations en santé. Pédagogie Médicale 2004;5:230-40.

33. McGaghie W, McCrimmon D, Mitchell G, Thompson JA, Ravitch MM, Quantitative concept mapping in pulmonary physiology: comparison of student and faculty knowledge structures. Adv Physiol Educ 2000;23-1:72-81.

34. Guide d'analyse de la littérature et gradation des recommandations. Paris : ANAES, 2000. [On-line] Disponible sur: http: //www . has-sante. fr.

35. Rendas AB. Toward meaningful learning in undergraduate medical education using concept maps in a PBL pathophysiology course. Adv Physiol Educ 2006;30:23-9.

36. Akinsanya MW. Concept Mapping for meaningful learning. Nurs Educ Today 2004;24:41-6.

37. Gonzalez HL, Palencia AP, Umana LA, Galindo L, Villafrade M LA, Mediated learning experience and concept maps: a pedagogical tool for achieving meaningful learning in medical physiology student. Adv Physiol Educ 2008;32:312-16.

38. All A, LaRae IH. Serial concept maps: tools for concept analysis. J Nurs Educ 2007;46-5:217-24.

39. Masmoudi S, Abdelassem F. TIC, du multimédia à la cartographie conceptuelle : cognition et modulation motivationnelle, in Naceur A, Masmoudi S. Cognition, Emotion \& Motivation : intégrer... mieux expliquer la performance, Tunis : Éditions du CNIPRE 2008;543-68. 
40. Marchand C, Crozet C, Martin D, Robert J-J, d'Ivernois J-F., Gagnayre R. Étude par cartes conceptuelles des connaissances sur l'alimentation des enfants diabétiques et de leurs parents. Revue Médicale Suisse 2007;3:868-72.

41. Michaud J-P, Marchand C, Blanco Pignat I, Ruiz J. Élaboration d'une carte conceptuelle en éducation thérapeutique avec un groupe de patients diabétiques : intérêts pédagogiques. Éducation du Patient et Enjeux de Santé 2008;26:12-7.

42. Pinosa C, Marchand C, Tubiana-Rufi N, Gagnayre R, Albano MG, d'Ivernois JF. The use of concept mapping to enlighten the knowledge networks of diabetic children: a pilot study. Diabetes Metab 2004;30:527-34.

43. Hinck SM. Student learning with concept mapping care plans in community-based education. J Prof Nurs 2006;22:23-29.

44. Taylor J, Wros P. Concept mapping: a nursing model for care planning. J Nurs Educ 2007;46:211-6.

45. Weiss L, Levison S. Tools for integrating women's health into Medical Education: clinical cases and concept mapping. Acad Med 2000;75-11:1081-6.

46. Srinavasan M, McElvany M, Shay JM, Shavelson RJ, West DC. Measuring knowledge structure: reliabilty of concept mapping assessment in medical education. Acad Med 2008;83-12:1196-203.

47. Schmidt HJ. Alternative approaches to concept mapping and implications for medical education: commentary on reliability, validity and future research directions. Adv Health Sci Educ 2006;11:69-76.

48. Mac Neil M. Concept mapping as a means of course evaluation. J Nurs Educ 2007;46:232-4.

49. Delorme F, Delestre N, Pecuchet JP. Evaluer l'apprenant à l'aide de cartes conceptuelles - Conférence internationale sur les techniques de l'information et la communication dans l'enseignement. TICE 2004, 25-31.

50. Daley B, Torre D. Concept maps in medical education: an analytical literature review. Med Educ 2010:44:440-8.

51. Marchand C. Les connaissances antérieures des patients sur leur maladie dans le contexte de leur éducation : analyse de leur rôle, de leur nature, de leur configuration cognitive et de leur évolution, par l'utilisation de cartes conceptuelles. Thèse de doctorat en sciences de l'éducation, Université Paris V, 2000.

52. Comte F. Existe-t-il une pédagogie du raisonnement médical dans les écoles de sages-femmes ? Quelques propositions pour le référentiel de formation. Mémoire pour l'obtention du master éducation clinique et santé, Université Paris 13, 2008.
53. Massart V, Frayens A, Giet D. Évaluation préliminaire de l'impact d'un dispositif d'apprentissage à la résolution de problème complexe (ARPc). Pédagogie Médicale 2008;9:141-56

54. Luckowski A. Concept mapping as a critical thinking tool for nurse educators. J Nurs Staff Dev 2003;19-5:225-30.

55. Ferrario C. Developing nurses' critical thinking skills with concept mapping. J Nurs Staff Dev 2004;20-6:261-7

56. Hill C. Integrating clinical experiences into a concept mapping process. Nurse Educator 2006;31:36-39.

57. Ellerman CR, Kataoka-Yahiro MR, Wong LC. Logic models used to enhance critical thinking. J Nurs Educ 2006;45:220-7.

58. Fonteyn M. Concept mapping: an easy teaching strategy that contribuates to understanding and may improve critical thinking. J Nurs Educ 2007;46-5:199-200.

59. Wilgis M. Concept mapping: an educational strategy to improve graduate nurses' critical thinking skills during a hospital orientation program. J Contin Educ Nurs 2008;39:119-26.

60. Kostovich C, Poradzizs, Wood K, O’Brien K. Learning style preference and student aptitude for concept maps. J Nurs Educ 2007;46:225-31.

61. Dewey J. Comment nous pensons. Édition originale 1986: How we think, traduit de l'anglais (USA) par Ovide Decroly, Paris: Le Seuil, Les empêcheurs de penser en rond, 2004.

62. Naccache N, Samson L, Jouquan J. Le portfolio en éducation des sciences de la santé : un outil d'apprentissage, de développement professionnel et d'évaluation. Pédagogie Médicale 2006;7:110-27.

63. Burke J, O'Campo P, Peak GL, Gielen AC, McDonnell KA, Trochim WMK. An introduction to concept mapping as a participatory public health research method. Qual Health Res 2005;15:1392-410.

64. Crevier F. Un modèle MOT vaut mille mots... Revue Internationale d'Ingénierie 2003;7:1-6.

65. Fournier JP, Demeester A, Charlin B. Script Concordance Tests: Guidelines for construction. BMC Medical Information and Decision Making 2008;8-18

66. Nesbit J, Adesope O. Learning with Concept and Knowledge maps: A Meta-Analysis. Rev Educ Res 2006;76:413-48

Correspondance et offprints : Anne Demeester, École Universitaire de Maïeutique Marseille Méditerranée, Campus Santé Nord, Bd Pierre Dramard, 13344 Marseille Cedex 15, France.

Mailto : anne.demeester@univmed.fr 